\title{
$\mathfrak{R}_{\mathfrak{m} \mathfrak{m a r k z}}$
}

oN

\section{IDIOPATHIC DILATATION OF THE COLON.}

BY HERBERT P. HAWKINS, M.D.Oxon., F.R.C.P., PHYSICIAN AND LECTURER ON MEDICINE AT ST. THOMAS'S HOSPITAL.

THE odd term " idiopathic dilatation of the colon" is well understood as applying to cases where the dilatation does not arise from any obvious mechanical obstruction inside or outside the bowel. The origin is mysterious. It is possible that these nine cases from St. Thomas's Hospital and some borrowed cases from other sources, if read together, may justify the belief that the condition at all ages arises through a congenital nervous defect. "Neuropathic dilatation and hypertrophy" becomes then a better name for the disease. But the striking point to $\mathrm{my}$ mind is the importance of an early diagnosis, because in every case there is a period, short or long, during which a cure can be effected by surgical treatment. The life, however, in all these cases is precarious, and the final hopeless toxaemic stage sets in so rapidly and unexpectedly that as a matter of fact nearly all published instances are taken from the dead, and very few recoveries are recorded. I doubt if the best method of surgical treatment has yet been worked out, but from what I have seen and read it seems probable that colotomy is useless, that excision of any part of the colon should never be permitted, but that the best result with the greatest safety is given by anastomosis of the iliac and pelvic colon, coupled with fixation of the dilated pelvic loop.

The picture of the condition in the fewest words comprises: (1) A history of constipation from birth or from the earliest recollection, which is compatible, however with fairly good health, until the final stage is at hand, the first sign of failure being often a loss of weight. (2) A constipation which often alternates with diarrhoea, and which at its worst is unlike that of obstruction, inasmuch as flatus is often passed and faecal matter may be drained away through a rigid rectal tube. (3) Abdominal enlargement, variable or constant, often asymmetrical, with prominence in the left iliac region, the abdomen, though distended, being seldom tense and often surprisingly flaccid, and rarely presenting any impairment of resonance. (4) Slow alterations in shape, especially in the left iliac region, observable only with patience, quite unlike the tense peristalsis seen in real obstruction. (5) The absence or rarity of pain and vomiting. Neither with real intestinal obstruction nor with simple non-dilating constipation should there be any confusion when this condition is far advanced. The difficulty lies in distinguishing its early stage from a simple constipation, and in recognizing the point at which the case must pass from physician to surgeon.

\section{The Origin of the Condition.}

(a) The Anatomical Factor

The old term "sigmoid flexure" may be discarded. The iliac colon lies in the left iliac fossa fixed to the iliacus. The pelvic colon begins at the inner margin of

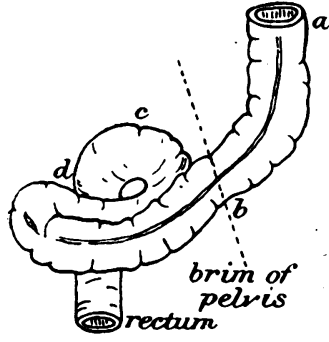

Fig. 1.-Normal.

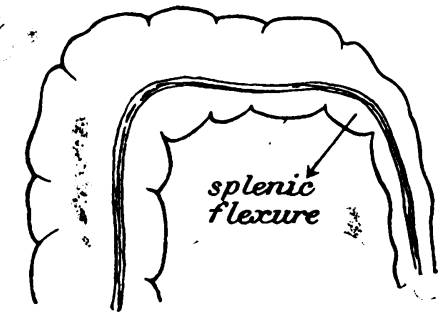

Fig. 5.-Case IV. the left psoas, dips into the pelvis, running for a variable distance to the right, then bends backwards and returns along the posterior wall of the pelvis to the third piece of the sacrum, where it joins the rectum. It is a loop provided with a mesentery, and is entirely free (Fig. 1, iliac colon $a$ to $b$, pelvic colon $b$ to $d$ ). In the adult it lies in the pelvis on the bladder or uterus. In the child at birth it is proportionately longer than in the adult, and it lies in the abdomen, only the lower end being contained within the pelvis. It varies greatly in length. Cunningham ${ }^{1}$ gives the average length as 16 or 17 in., with extremes of 5 and 35 in. If the loop is unusually long, it is variously convoluted, and its butt-end may often be found in the right iliac fossa. In nearly all cases of this disease the dilatation begins below in the lower 3 in of the pelvic colon, at the spot where the mobile and mesentery-provided bowel begins to lose mobility and mesentery, and merges into the fixed rectum. So constant is the starting point at this place of transition that there is temptation to consider that the anatomical arrangement is alone responsible for the dilatation, and various theories of "valve formation," "folding," and "kinking" have arisen. The valvular theory (Roser) is founded on the undoubted fact that even in advanced stages of the disease, when the bowel no longer responds to purgatives or enemata, the passage of a rigid tube 8 or $10 \mathrm{in}$. from the anus results in a drainage of faeces and gas, which ceases at once when the tube is withdrawn. On the strength of this, treatment has been attempted in some cases by leaving a long tube in the rectum. But this drainage by tube is easily understood when the parts are examined after death (Figs. 2 and 7) without presupposing the existence of a valvular obstruction, which I think has never been demonstrated. Certainly there is no structural narrowing of the bowel. In fact, the passage right down to the anus is perfectly wide and free, even in cases which have ended fatally, and it is marvellous (Figs. 3 and 7) that the escape of faeces and gas has been so scanty and imperfect. As regards the theory of an obstructive folding or kinking from a superfluity of the pelvic colon, it is met with the fatal objections (1) that in the post-mortem room long and much folded, but healthy, pelvic colons are very common, while this peculiar dilatation is rare; (2) that in some fatal cases of this dilatation it is clear that the pelvic lonp was short and not folded; (3) that the commencement (that is, the lower end) of the dilatation is gradual and not abrupt, as would have been the case if the bowel had been kinked at this spot; and (4) that the signs and symptoms of the condition are wholly different from those which are met with in any form of intestinal obstruction.

It may be allowed, perhaps, that the anatomical arrangement is in some degree responsible for the dilatation But it seems to be equally clear that a purely anatomical explanation will not suffice. There must be another factor in its production, and it is difficult to conceive that this missing factor can be anything but a defect on the nervous side, especially if, as I believe, the condition may actually be present in utero before ever the bowel has begun to act.

\section{(b) The Nervcus Factor.}

A neuropathic origin admits of no proof. It is probable that Hale White's ${ }^{2}$ expression "congenital inertness of the colon" takes us as far as we can go into the mystery. If by "inertness" we mean a neuro-muscular defect, through which a section of the colon, though it opposes no obstacle, is yet (continuously or from time to time) incapable of forwarding its contents, I believe we have in this the basis of the disease. I think we may realize a difference between a physiological and a physical_obstruction of the bowel.

The evidence seems to show that this primary defect in every instance, child or adult, is truly congenital, and that the dilatation is not acquired by faulty habits or abuse of aperients and enemata. I think that these cases should be regarded as extreme and persistent examples of $a$ common form of constipation in children, and not as constituting a separate type of disease. Many children are constipated from the day of birth, at so early a point in their career that no error in diet can be held as the cause, and it is reasonable to consider that this constipation is due to a congenital condition, be it called inertness of the colon, a neuro-muscular defect, or a lack of peristaltic response to normal stimuli. These are the constipated children, in whom the insertion of the finger or a piece of soap into the rectum will often produce a better result than an aperient. As these children grow older, nearly all of them regain, or rather acquire, a normal responsive and expulsive power before any dilatation sets in. A small number of these relapse at later stages, [2409] 
and here, I think, belong most of the cases described by Cheadle ${ }^{3}$ in some of which evidence of dilatation can certainly be seen, although (perhaps with one exception) they were still within the limits of recovery. In one of his cases puncture of the colon was necessary, and speaking generally they seem to indicate the last stage at which medical treatment can be continued with success. In a few instances, however, out of this multitude of constipated infants dilatation sets in, and, more than that, it persists. Even then a compensatorg hypertrophy may carry the child comfortably through some months or years of life, constipated but not ailing, until the final failure brings him before us, generally in a hopeless state, and he is scattered in small numbers through medical literature under the label of idiopathic dilatation of the colon.

Further, I see no reason for considering that the colondilatation of the adult differs in origin from that of the child. It is certainly odd that instances of the condition are not uncommon up to the age of 10 , are very rare from 10 to 40 , but appear again, though still uncommonly, from 40 onwards. There is thus a curious age-gap. It is odd also that at an early age males are almost exclusively affected, while at a later age instances occur in males and females alike. But I do not believe that these is any reason either in this age-gap or in this sex-differentiation for dissociating the child's disease from that of the elderly. My instances of all ages seem to hang together as originating in the same way. History, symptoms, anatomy, and fate are the same. I think it may be said that the primary nervous defect may produce its outward and visible sign of colon.dilatation sometimes even in utero, commonly soon after birth, but that if the individual survives the developmental period he usually tides through adolescence and early adult life, only, it may be, to succumb to dilatation in later years when the additional influence of age comes into play.

There are indications that the nervous element in the causation is generally paralytic, but it is (cf. Case IX) sometimes spastic. A purely nervous origin is no new theory. About 1880 Hilton Fagge, called to see a child, aged 12 months, suffering with constipation and abdominal distension from birth, gave it as his opinion that the cause was "spasm of rectum." The child died three years later, and the condition was reported by Gee." At the post-mortem examination the pelvic colon looked like a stomach. It lay across the hypogastrium, extending into the right iliac fossa. Below this dilated part the rectum, and perhaps the lower end of the pelvic colon, showed a spastic contraction "but not a very tight stricture, easily admitting the index finger."

Morbid Anatomy.

The condition of the parts is best studied from below upwards. The anus is normal in structure, but cases are on record where anal spasm, which is certainly a factor in the constipation of children, has been probably the sole cause of this colon-dilatation. I believe that an instance reported by Fenwick ${ }^{6}$ gives us the key to the understanding of all these cases. Here the dilatation which affected the whole colon extended right down to the anus, and the rectum was funnel-shaped, being $5 \mathrm{in}$. in circumference at its junction with the pelvic colon, but only $2 \frac{1}{2}$ in. round just above the anus. The agency of anal spasm can hardly be doubted. This funnel-shape is nearly always present, and I think that the majority of cases differ from Fenwick's case only in the fact that the funnel is found in the pelvic colon and not in the rectum. $\mathrm{As}$ a rule the rectum is normal, but sometimes it is decidedly roomy, as if at one time or another there had been anal spasm. In most cases the dilatation begins above the rectum, but the lower two or three inches of the pelvic colon often remain unaffected. The enlargement is rapid, but it is never abrupt. The maximum dilatation is often reached in a distance of three or four inches, and this section of the bowel may be likened to a funnel, or to the neck and shoulder of an inverted bottle (Figs. 3 and 7). The size reached may be enormous. A diameter of 6 in. is not uncommon; one of 8 in. has been recorded. In Case viI (Fig. 8) the circumference after puncture was 19 in. As a rule the loop shape of the pelvic colon is preserved, and this part rises entirely out of the pelvis, the anterior surface of its mesentery becoming posterior, with the butt end of the loop lying at or above the umbilicus, and sometimes actually under the right costal margin.
On opening the abdomen it may be found to conceal every other structure, except, perhaps, part of the transverse colon. The two nearly parallel limbs of the loop may sometimes be seen through the abdominal wall, as in Case II (Fig. 4). Sometimes, probably in cases where the pelvic colon was originally short, the dilated bowel is more of a bag than a loop. It may then resemble a stomach lying right across the hypogastrium, with its convexity in the right iliac fossa. In a case recorded by $\mathrm{Gee}^{6}$ an odd movable lump in the right iliac fossa was found post mortem to be a faecal mass in the pelvic colon. Occasionally the lower part of the right limb of the loop sags down into the pelvis, becoming pouch-like, as in Case I (Fig. 2), and in this case an evacuation of the bowel must be of the nature of a passive overflow from a reservoir.

As regards the rest of the colon, the condition varies. Occasionally it is normal in all respects, and the dilatation subsides as the pelvic colon merges into the fixed iliac colon. The upper end of the pelvic loop or bag is then shaped like the lower end. I think this pure pelvic dilatation is uncommon, and occurs only in young children. Langmead ${ }^{7}$ records an instance with associated tetany. Far more commonly the rest of the colon is also affected in some degree. Often the dilatation reaches to and fades away in the iliac or descending colon. But in some cases it is continued uniformly onwards up the descending colon, and ceases gradually in the transverse or ascending colon, only in rare instances reaching the caecum. In these extreme cases the abdominal distension is by itself a danger to life. Interference with respiration is the common result. In rare cases hydronephrosis has been noted, and occasionally oedema of the lower extremities due to pressure on iliac veins. Tetany has been known to occur, and, as in the case of dilatation of the stomach, it requires immediate operation.

The condition of the small intestine can be described in absolute terms. It is never dilated. During operation and after death it is found to be small, collapsed, empty, and grey, and it is generally gathered into an inconspicuous central handful lying on the vertebral column buried beneath the colon.

The dilatation of the colon is not a matter of recent distension by gas. It is clearly the work of time, for in nearly every case there is great hypertrophy of muscle. The dilatation and hypertrophy are associated together from end to end of the affected part of the bowel. One cannot doubt that the hypertrophy is a secondary compensatory development, an Arbeitshypertrophie. In cases in which life is prolonged for many years, no doubt this hypertrophy is for a time sufficient to counterbalance the paretic or spastic section of bowel below, but a relative insufficiency must set in sooner or later. The circular muscular coat may be three times as thick as the longitudinal taenia, and the whole wall may have a thickness of $\frac{1}{4}$ in. The bowel is thus rendered abnormally rigid, and on puncture it shows very little tendency to collapse. In Case VII, however, an extreme instance of dilatation of the whole colon, there was certainly no hypertrophy at the time of death, and in Langmead's case $^{8}$ the slight thickening of the pelvic colon was found microscopically to be due to hypertrophy of the muscularis mucosae alone.

The mesentery of the pelvic colon in these cases is sometimes described as being thickened. I believe this change is uncommon, but it was present in Case v. There are no adhesions. Ulceration, such as commonly occurs in long-continued obstruction of the colon, is very rare in this disease, but it has been noted in some cases, and one ulcer was present in Case Ix. In one recorded case perforation occurred. During life, and in the post-mortem room, the faecal matter in the dilated colon is nearly always semifluid. It often has a curious deficiency of faecal smell, and I have never found it to be offensive. The colour is usually yellow or green, or greenish-yellow. But the contents of the colon are mainly gaseous, and it is in consequence of this disproportion between the amounts of gas and faeces that the abdomen in these cases is generally resonant all over its surface. The gas pressure must be regarded as the actual dilating force.

The Fully-developed Condition in Chindren. The following cases are examples of the fully-developed condition in children : 
CASE 1.-Defective Action of Colon from Birth, but fairly good Health: Dilatation and Hypertrophy of greater Part of Colon: Colotomy : Death.

Boy, aged 12 months, admitted under the care of Dr. Sharkey. "From birth" there had been unusual difficulty with the bowels, mainly constipation with occasional bouts of diarrhoes. Purgatives had been in common use; otherwise he had been fairly well. One week before admission into hospital his mother noticed in his abdomen " 8 lump that seemed to move," presumably peristalsis in the pelvic colon. At that time his bowels had not been open for five days, but there was no vomiting and no appearance of pain. On examination he was well nourished, but extremely ill. The abdomen was greatly distended but resonant everywhere, and indications of a large pelvic colon could be seen. An exploratory incision was made by Mr. Sargent at once, and the nature of the case being recognized, colotomy was performed. No improvement followed and the child died on the next day with a temperature of $106^{\circ}$.

Post mortem it was found that the anus and rectum and lower 3 in. of the pelvic colon were normal. At this point dilatation and hypertrophy began, the greatest diameter (about 6 in.) being reached in a distance of 4 in., the huge resulting pelvic loop filling the lower half of the abdomen. The righthand part of the loop had sagged down into the pelvis (Fig. 2). The iliac, descending, and transverse colon was also greatly dilated and hypertrophied, but these changes gradually disappeared in the ascending colon, and the

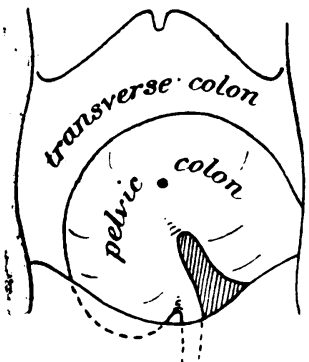

Fig. 2.-Case I

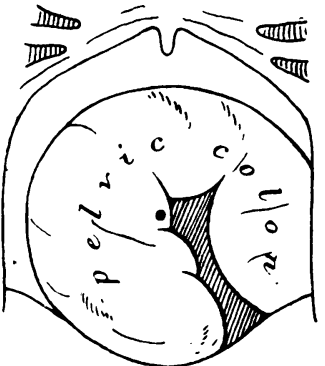

Fig. 8.-Case VII. caecum was normal. The whole colon contrined soft yellow faeces. When this with some pressure was squeezed out faeces. When this with some pressure was squeezed out freely from the caecum through the whole length of the bowel out at the anus, and it was difficult to believe that there could out at the anus, and it was difficult to believe that there could have been any impediment to the outflow of the soft contents
during life. Fig. 3 shows the appearance after removal and preservation.

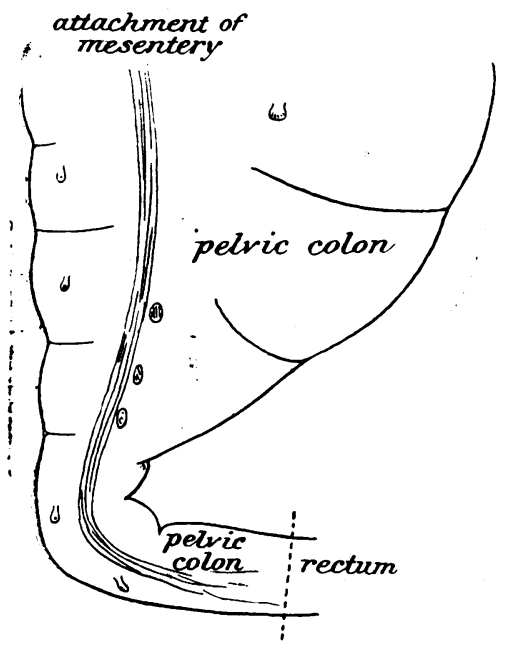

Fig. 3.-Case I.

The history in this case seems scanty but it is probably complete. Constipation there had been from birth, but it had attracted little attention and the general health had been good. We may conclude that hypertrophy had kept pace with the increasing dilatation during the greater part of the twelve months of life. Fairly good health for a variable time, ending in rapid failure of compensation, stagnation, toxaemia, and death, is a common story in these cases. Other points to be noted are the absence of pain and of vomiting. These are rarely present, and their absence serves to distinguish the condition clinically from any form of obstruction. The so-called diarchoea, which is a passive overflow, is common enough. The terminal pyrexia is noted in other cases.
The same history, symptoms, and signs appear in Case II though the end was reached more gradually. The usual terminal wasting was present. This case shows the com mon condition of the abdomen, which is one of distension with flaccidity or doughiness, so that the examining hand sinks in.

CASE II.-Defective Action of Colon from Birth: Dilatation of Pelvic Colon : Death.

Boy, aged 10 months. He had always been weakly. "From boy, aged 10 months. He had always been weakly. "From birth" the bowels had never acted without aperients and
their result was not satisfactory; he had been breast-fed but their result was not satisfactory ; he had been breast-fed but
was now weaned. He was now extremely thin, the miserable was now weaned. He was now extremely thin, the miserable
limbs and wizened face contrasting with an enormous abdomen. The abdominal wall was very thin, and much of the disposition of the intestine could be seen. The whole abdomen was distended, but two large coils were always seen running up from the left ilise fosss, and converging under the right costal mare (Fig. 4.) It can hardly be doubted that this was the pelvic

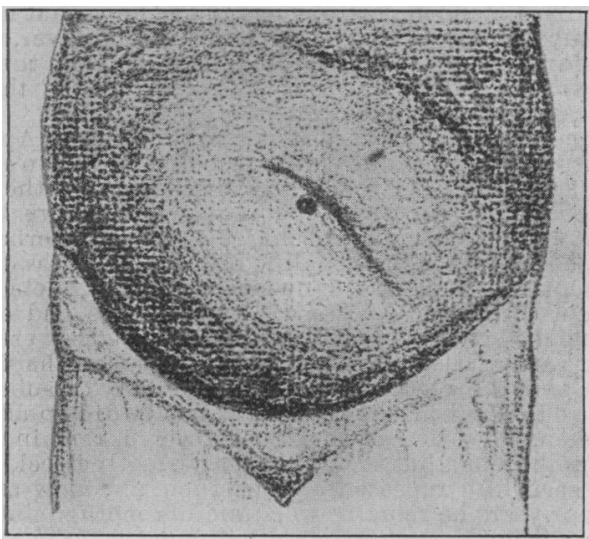

Fig. 4.-Case II.

colon. No peristalsis was observed, the dilated bowel being completely deprived of motor power. The anus and rectum were normal. There was no sign of pain, and no vomiting. For ten days enemata and aperients produced little gushes of thin faecal fluid, the child wasting and losing ground with a rising temperature, which was $104^{\circ}$ at death. Surgical treatment did not seem possible. A post-mortem examination could not be obtained.

The next case is another example of the same story, but it is of more interest, because the disease was less advanced, and the condition permitted surgical treatment and a relative cure. As in Case $I$, this boy had enjoyed fairly good health. All his life aperients had been used from time to time, but his mother had not thought that he differed from other boys in this respect. It is likely, however, that there was some want of observation on the part of the mother, for the history shows that a full year before his admission into hospital his distended abdomen had attracted attention, and yet on his admission she was not even then aware of his peculiar appearance in this respect. The abdomen in this case was at first tense, but it subsequently became flaccid, although it retained its former large dimensions. The impairment of resonance found here is unusual.

CASE III.-Early History of slight Constipation: Dilatation of Pelvic Colon: Anastomosis of Iliac and Pelvic Colon: Recovery.

Boy, aged 7 years. At the age of 2 he was treated at the Boy, aged 7 years. At the age of 2 he was treated at the
Eveling Hospital for rickets. When he was nearly 6 years old Evelina Hospital for rickets. When he was nearly 6 years old
he had tonsils and adenoids removed at St. Thomas's Hospital. As he was noticed to be thin, and the abdomen was then so prominent as to attract attention, he was transferred to the medical side for treatment, but the nature of the abdominal enlargement was not recognized, and he ceased to attend. Fourteen months later he was run over by a cart and had three ribs broken and this was probably a very fortunate accident. On admission into hospital on account of the injury the abdoperis a men was found to be greatly distended ; some. slow, gentlo peristaltic movements could be seen ; and it required strong aperients and enemata to get any action of the bowels. There was uniform distension of the abdomen, which measured $27 \frac{1}{2}$ in. at the umbilicus, but the flanks especially were bulging. It was resonant everywhere except in the flanks and the left lower quadrant, and in this latter region there was a vague sensation as of $a$ rather tense rounded swelling under the hand. The abdominal skin was tightly stretched and shing, but showed no veins. The thoracic organs were pushed upwards. The subcostal angle was wide and the costal margin everted. The rectum was large and empty. In the fortnight 
after admission only three actions of the bowels were obtained by enemata and large doses of castor-oil and other pargatives. The impairment of resonance and the feeling of an ill-defined rounded swelling in the left lower part of the abdomen remained constant. Moreover, although the whole abdomen was tensely distended, slow peristaltic movements coald be seen in the left iliac fossa, and frequent borborygmi could be heard the left iliac fossa, and frequent borborygmi could be heard at a considerable distance from his bed. No doubt was felt as good health. He was in no discomfort, was not sick, retained a good appetite, and cared not at all whether his bowels were open or not.

It did not appear to be worth while to embark on a course of medical treatment. My colleague Mr. Makins, therefore opened the sbdomen by a 4 in. incision below the umbilicus to opent of the middle lin. that came into view was greatly distended, and the pelvic that came into view was greatly distended, and the pelvic colon was found to be a bag rather than a loop extending up to the umbilicus. He made a lateral anastomosis betwoen the lower ends of the iliac and the pelvic colon, thus giving a bag, and this distended part was left in the hope that it might undergo subsequent atrophy. It was felt, however, that a complete cure was not to be expected, because from the physical signs it seemed probable that the greater part of the colon was also in some degree affected

For some weeks there was little improvement. Aperients were necessary, magnesium sulphate being found to give the best result. There was frequently distension of the shortcircuited pelvic bag, for which the passage of a long rectal tube usually gave relief with the discharge of flatus. Perhaps as a consequence of the tension, healing of the wound was delayed and it eventually suppurated, opened up in its whole length, and was not completely closed till two months had elapsed. But during the latter part of this time the bowels were acting in a much more satisfactory way, aperients were hardly ever necessary, and the rectal tube was discarded. A subsequent course of abdominal massage and electricity did much good. course of abdominal massage and electricity did much good. (23 in. at umbilicus), but it was always perfectly flaccid.

I have seen him repeatedly since then, and now after the lapse of four years he remains well, and his mother states that an aperient is not necessary more than once or twice a month Bat his pelvic bag is still in existence, and the occasional appearance of its outline when partly filled with gas shows that its size has not undergone much diminution. Its contents, however, are apparently purely gaseous, and it gives rise to no discomfort. Judging from Case viII, there is, perhaps, some risk of volvulus.

Examples of a Possible Early Stage.

I think it is possible that the next case may represent the very earliest stage of the condition. There is evidence here of a varying paralytic distension of parts of the colon, which I think was certainly present in utero, produsing immediately after birth vomiting, constipation, and diarrhoea. It is conceivable that, if the child had lived, permanent dilatation and hypertrophy would have arisen The terminal pyrexia may have been of pulmonary origin.

CaSE IV.-Defective Action of Colon from Birth, but Colon found normal Post Mortem : Constipation: Diarrhoea : Vomiting: Wasting : Death

Boy, aged 3 weeks. He was born prematurely. It was noted at birth that the abdomen was unusually large, and the grandmother volunteered the statement thet "none of the black mother volunteered the statement that "none of the black stuff that babies pass "had appeared (compare similar statement in Osler's case). During the first week he took the breast proved, but he vomited nearly every feed, as a rule without delay. The bowels had never acted satisfactorily, there being either constipation or, after aperients, the passage of small liquid stools.

On examination, the child was wasted and very feeble, weighing $7 \mathrm{lb} .9 \mathrm{oz}$.' The abdomen was considerably distended, but not tense. The epigastrium was much more prominent than any other part. Abdominal respiratory movement was than any other part. Abdominal respiratory movement was good, and there appeared to be no pain or tenderness. There was no sign of disease elsewhere. From this history of vomiting and these physical signs, it seemed probable that examination I was surprised to find palpable and visible signs of considerable distension of the colon. The distension clearly affected the caecum and ascending colon and to a less degree the part lying in the left iliac fossa, and it seemed not unlikely that the epigastric prominence already mentioned was, in part at any rate, due to a distended transverse colon. No active movements such as occur in complete intestinal obstruction were seen at any time but on prolonged observation the prominent parts showed slow alterations in shape. In comminent parts showed slow alterations in shape. In comparison with the distension, the abdomen was surprisingly but to the touch the whole abdomen resembled a thin-walled, half-inflated airbag. The anus and rectum were normal

Four days later the physical signs remained unaltered. During this period the child continued to vomit occasionally. Calomel was given on the first day, and thereafter three or four times a day a small liquid stool drained away. The stools were by no means offensive. The temperature was rising gradually, and on the fourth evening reached $104^{\circ}$.

With the possibility of some malformation about the junction of colon and rectum before us, Mr. Makins at my request made an exploratory incision into the abdomen sufficient to show if any condition was present which could be remedied. The caecum, ascending and transverse colon, were greatly distended, the distension ceasing abruptly about the splenic flexure. The change from distended to normal bowel occupied about $\frac{1}{2}$ in., and it was clear that there was no obstructive cause for the distension. The distended part was abnormally hyperaemic, and contrasted with the rest of the colon, which was pale. From the splenic flexure downwards the colon was apparently of normal size. The small intestine was empty, collapsed, and grey. The stomach was of normal size, and the pylorus showed no abnormality. Fig. 5 is a diagram of the colon. On the next day the temperature rose rapidly, and
death took place two days later without any change in the death took place two days later without any
condition, the thermometer registering $109^{\circ}$.

Post-mortem examination showed some collapse and broncho-pneumonia in the lower lobes of both lungs, this condition being probably the immediate cause of death. There was no sign of disease of stomach or intestine. There was nothing to account for the distension of the colon, which had resumed its normal dimensions and appearance. There was a very large intussusception high up in the small intestine formed just before death. The abdominal incision was healthy, and there was no peritonitis.

In this case there was no appearance of pain, and the whole picture suggests a pure paralytic condition. A similar case has been reported by Dr. Dodd of Hove, which takes us a stage further, inasmuch as the dilatation, which was of longer standing than in my case, persisted after death. The child lived for three months. The conditions found post mortem are suggestive of a spastic as well as a paralytic state; signs of abdominal pain were seen, and the curious state of the stomach is noteworthy in this connexion, making one reflect on the possible origin of hypertrophic stenosis of the pylorus. Dr. Dodd's case shows evidence of defective action of the colon from birth, of distension and spasm, with straining, constipation, pain, vomiting, and wasting.

In this case, a male infant, there had been noticed by the nurse, "from the time of birth," an unusual straining at stool. At first the bowels were open once or twice every day, and later once every other day. For the first three weeks of life the child throve. At the end of the third week vomiting began, usually occurring five or ten minutes after taking the breast, and in a few days it seemed to be returning the whole feed. There was the appearance of pain, and the child lay curled up, with thighs flexed on abdomen and feet crossed, crying out at short intervals. After temporary improvement the vomiting, pain, and constipation recurred. Examination under chloroform revealed nothing abnormal. Aperients and enemata gradually became useless; abnormal. Aperients and enemata gradually became useless; anything that was passed looked like yellowish-brown oil-
paint. Wasting was rapid and the child died at the age of paint. Wasting was rapid and the child died at the age of
12 weeks. At the post-mortem examination the ascending and transverse colon were found to be little larger than an ordinary lead pencil. The head of the caecum was normal. The descending colon, iliac, and pelvic colon and rectum were distended, with some partial annular contractions of the pelvic colon. The small intestine was collapsed. It is interesting to

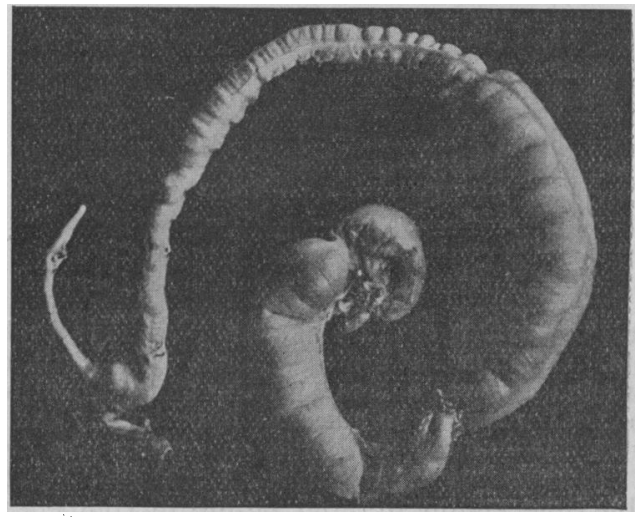

Fig. 6.

note that there was a sort of hour-glass constriction of the stomach near the cardiac end, apparently due to spasm. There was no disease elsewhere. The condition of the colon is well shown by the photograph (Fig. 6) which Dr. Dodd has kindly allowed me to use. The constriction seen at the end of the descending colon is artificial. 
Another case is recorded by Osler. ${ }^{10}$ There is here the same. story of colon defect from birth, with vomiting, pain, and constipation. The condition, however, was kept under control by the use of a rectal tube, though there was no real improvement, and the later history is not reported.

A male child, aged 7 months, came before him with the history that it had been noticed from birth that the "napkin were not soiled." Otherwise its health was good. The abdomen soon after birth becsme swollen and tense, but by means of a catheter some black, tar-like faeces were brought away. From this time up to admission into hospital the child had had only five or six natural evacuations, and injections had been necessary. The child was at first well-nourished, but latterly had been The child was at first well-nourished, but latterly had been abing weight. If the injections were given regularly, the seem ill. If the in jections failed, the abdomen became distended, vomiting began, and there was the appearance of pain. On examination, the abdomen was greatly distended and ver tense, the costal margins and ensiform cartilage being everted Intestinal coils were visible, particularly one very large coil between navel and ensiform cartilage. The mother had noticed peristalsis. The anus and rectum were normal. A catheter was passed without difficulty, and when it resched a distance of $8 \mathrm{in}$. from the anus much gas escaped, and fluid distance of $8 \mathrm{in}$. from the anus much gas escaped, and fluid faeces also if there had been given (as was the mother's custom) a previous injection of water. The abdomen would become child remsined in the same state, the long tube and injections being in constant use. In relation to this case, Osler sug gested that "here we may possibly have an illustration of the early condition which leads ultimately to the enormous dilatation and hypertrophy met with in such cases as that of the man reported by Formad," who lived to the age of 23 , constipated and distended from birth, with a colon at death from 15 in. to 30 in. in circumference. "From birth there seems to have been an inability in the large bowel to empty

The next case seems to have been of the same nature, but it differs from the preceding instances in that the colon condition, presumably paralytic and consequently painless, was intermittent, and the bowel apparently recovered between the attacks, so that the boy had reached the age of 5 alive.

Cask v.-Intermittent, patchy Distension of the Colon: Abdominal Exploration: Apparent Recovery.

Boy, aged 5 years, admitted under the care of Dr. Acland. His early history is not very full, but on two previous occasion he had been very ill with an attack like the present one. His present illness began with romiting and distension of the abdomen without pain, but the bowels continued to act. With no more urgent symptoms than these he soon became extremely ill, and he was sent up to the hospital as suffering from intestinal obstruction three weeks after the onset. On admission he had the face and sunken eyes which one associates with acute abdominal disease, his pulse-rate was 128, and he was so ill that infusion was necessary before anythin and he was so ill that infusion was necessary before anything
else could be done. The abdomen was moderately distended moving with respiration, resonant everywhere, presenting no other sign of disease than fullness and dullness in the flanks. An exploratory incision was made by Mr. Sargent. The small bowel was found to be shrivelled and collapsed. The upper part of the ascending colon and the hepatic flexure and the pelvic colon were much distended without any apparent canse, while the colon between these parts was of normal size. The condition, in fact, was much the same as in Case IV. Though he remained ill for some time he gradually improved, the remained ill for some time he gradually improved, the vomiting ceased, and he left hospital three weeks later, been unable to trace his subsequent career.

\section{Examples of the Fully-developed Condition in} ADurts.

The next case gives an example occurring in middle life. Except in these two points of greater age and of the sex, I do not see any reason to suppose that it differs from the preceding cases. The condition of the pelvic colon differed from that found in Case $I$ only in that it was more funnel-shaped and less pouched. It appeared that the trouble with her bowels dated from childhood at any rate. As in Case I, fairly good health had been enjoyed until the final collapse set in. It seems to have been a pure paralytic condition, with considerable hypertrophy.

CASE VI.-Defective Action of Colon from Infancy : Dilatation of whole Colon, especially the Pelvic Part: Incision of Bowel: Death.

Womsn, aged 39 . Her story of constipation went back to her earliest recollection. She was always in trouble in this respect but she thought that matters had been worse in the last four but she thought that matters had been worse in the last four
years. Nevertheless she had had fair health and borne eight children. Latterly, with bowels acting in some degree about once a week, she had begun to fail, and three weeks before admission she had taken to her bed. On examination she was seen to be thin and pinched. Her mental state was curious. She was slow, dazed, and apathetic, as if under the influence of a narcotic. The abdomen was greatly distended, but it was not quite symmetrical, the left side being more prominent than the right. It was resonant everywhere. There was a faint mapping-out of coils of intestine and slow alterawas a faint mapping-out of coils of intestine, and slow alterations could be seen in them, with occasional borborygmi. Though the abdomen was so large, it was singularly flaccid, and in this respect it was quite unlike the abdomen of intes tinal obstruction. Moreover, there was no pain or tenderness, and she was not sick. The rectum was normal. The abdomen was opened at once, the dilated pelvic colon was incised, and a large quantity of yellowish liquid faeces was removed, but it was not thought advisable to attempt anything further. Bhe died on the next day.

At the post-mortem examination the condition was nearly identical with that found in Case passed gradually at the upper part of the sacrum into a huge pelvic colon, which even now, though it had been nearly emptied at the operation on the previous day, filled the lower emptied at the operation on the previous day, filled the lower
half of the abdomen. It was almost entirely extrapelvic. There seemed to be no reason why its contents should not have poured into the rectum. Fig. 7 is a diagram of the parts. The

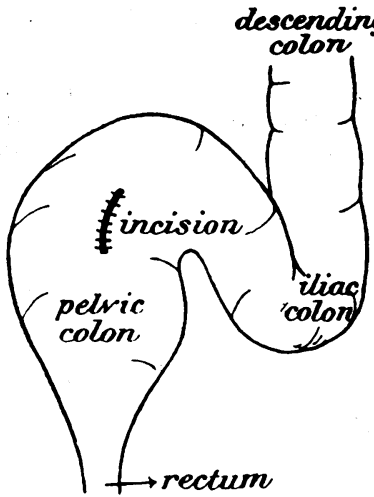

Fig. 7.-Case vi.

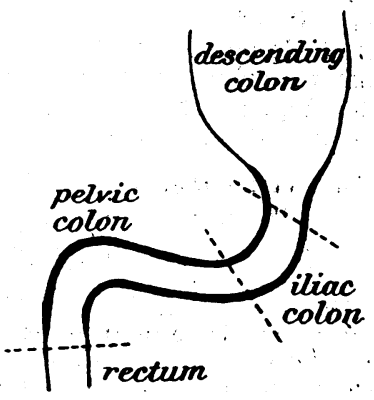

Fig. 9.-Case $1 x$. mesentery of the pelvic colon was apparently thickened. The whole colon was somewhat dilated, but the change gradually disappeared before the caecum was reached. Its wall was thickened, apparently from true muscular hypertrophy. The small intestine was collapsed and grey. "There was no sign of change inside the colon.

The next case is another example of the middle-life condition in a male. It differs only from the preceding case in the absence of any thickening or hypertrophy of the colon wall. I suppose it is possible that a late and final distension of the colon had obliterated all trace of a previous hypertrophy. There is here, as in other cases, a history of good health in spite of the dilatation, with the usual sudden collapse at the end.

CASE VII.-Defective Action of Colon from an Early Age Fairly Good Health: Dilatation of whole Colon: Death Man, aged 40, admitted under the care of Dr. Sharkey with the history that his bowels had never acted properly within his recollection. He had tided along for forty years, with the help of purgatives and enemata, in recent years relying almost entirely on the latter. He had worked up to a month before admission, and he had enjoyed fairly good health $\mathrm{He}$ had hardly ever been sick, had no abdominal pain, and had had a good appetite, and, in fact, the night before admission he consumed a pint of periwinkles.

On examination the face and limbs showed wasting. The abdomen was greatly distended, its greatest circumference being $43 \frac{1}{t}$ in.; some veins were visible, and indications of intestinal coils could be seen. No peristalsis was observed Percussion geve a trmpan custo note everywhere, and the whole obliterated. The rectum was large and empty. Enemats and castor oil gave no result, and puncture of the intestine was castor oil gave no result, and puncture of the intestine was
useless. He slowly sank, and died four days after admission. useless. He slowly sank, and died four days after admission. umbilicus was found to be 49 in. On opening the abdomen only the colon was seen, the pelvic loop taking nearly half the space, the rest being occupied by the transverse colon (Fig. 8). The gas in the pelvic colon was at a high pressure, and issued with a whiz on puncture, the bowel collapsing, but only down to about three-quarters of its original size. The gas had no faecal odour. The anus and rectum were normal. The dilatafion began, as usual, rather gradually just above the rectum and in the length of 4 in. the normal lower end of the pelvic and in the length of 4 in. the normal lower end of the pelvic measured 19 in. in circumference, even after the bowel had been punctured. It was entirely extrapelvic. There was no manner of obstruction, and there seemed to be no reason whatever why faeces should not have entered the rectum. 
The whole colon was dilated, the degree of dilatation diminisb. ing progressively upwards, the caecum being hardly affected. There was certainly no muscular hypertrophy. If there was any change, the colon wall was thinned. There was no appearance of disease of the mucous surface. The colon contained more than $31 \mathrm{lb}$. of greenish semifluid material like cooked spinach, having hardly any faecal odour. The small intestine was collapsed and empty. The upward thrust upon the diaphragm was extreme.

The next case is an example of the same kind, but she came under my observation at a time when there was a possibility of surgical treatment, and I think that after a rather hazardous career, she now has a good prospect of living out her days. The story is identical with that of the case recorded by Clutton, ${ }^{11}$ in which he excised the sigmoid flexure successfully. This cas further illustrates the risk of volvulus that is entailed if after the making of a short circuit, the pelvic loop is left free in the abdomen. In her case this accident happened twice. It illustraies also the fact that the inherent weakness of the colon and its consequent inability to withstand the distending force of contained gas will still persist even after excision of the greater part of the pelvic loop. Here, without any trace of obstruction, and in spite of the fact that the bowels continued to act fairly well eighteen months after excision of nearly the whole pelvic colon, a new loop some 9 in. in length was developed out of the 3 or 4 in. of the pelvic colon that remained below the point of anastomosis (Fig. 1, $c$ to $d$ ).

CASE VIII.-Defective Action of Colon from an Early Age: Dilatation of Pelvic Colon: Anastomosis: Recovery. Later Volvulus of Dilated Loop: Operation: Resection. Fixation: Recovery.

Woman aged 46. She had been constipated all her life. As a young girl she used to get two or three actions of the bowels in a week, one way or another. From the age of 20 to 40 in a week, one way or another. From the age of 20 to 40 regularly; she used to wait patiently and an action came regularly ; she used to wait patiently and an action came sooner or later, and this often without an aperient. During the last five years there had been increasing difficulty. Purga-
tives and enemata were frequently used. Occasionally when things were at their worst there had been some abdominal discomfort, but she had never been sick. The abdomen from time to time had become enlarged, and this condition had tended to become constant during the last year. She had nevertheless had good health, and had worked hard as a dressmaker.

On first admission she was certainly thin. The abdomen was considerably distended, but as in other cases it was curiously soft, so that the examining hand sank in, as into a feather bed. It was resonant everywhere. It was generally more prominent in the left lower quadrant than elsewhere, and sometimes this part stood out and exhibited slow gentle alterations in shape, so that the diagnosis admitted of no doubt. The rectum was normal and not large. She was a placid individual and felt no anxiety as to her future. With enemata and aperients and dieting there was some improvement, and she gained $5 \mathrm{lb}$. in weight in three weeks. But there was no she gained $5 \mathrm{lb}$. in weight in three weeks. But there was no
happy mean. She was either completely constipated or she passed eight or ten small liquid stools a day, clearly by overpassed eight or ten small liquid stools a day, clearly by over-
flow from the dilated pelvic colon ; and it seemed improbable that anything further was to be gained from medical treatment.

Mr. Makins therefore took her in hand at my request. Incision revealed a pelvic loop of which the upturned butt end was situated above the umbilicus, and an anastomosis was made between the iliac colon and some point low down in the pelvic colon below the dilated part, as in Case III. The result was quite satisfactory, and though the gas-containing loop remained in evidence the bowels acted fairly well. Three
months later, however, she was readmitted into hospital with - months later, however, she was readmitted into hospital with and completely resected, so that she was now provided with a short, fairly straight passage from iliac colon into rectum without any lateral cul-de-sac.

She now resumed work and remained well for eighteen months, having no difficulty in keeping her bowels open with an occasional mild aperient. She was then readmitted with a second volvulus. It was found at this third operation that the small portion of pelvic colon (Fig. 1,c-d) between the the small portion of pelvic colon (Fig. 1, c-d) between the anastomosis and the rectum had become dilated into a loop nearly as large as the original loop that had been resected.
This had become twisted with one and a half turns from left to right. The position of the loop was rectified, and she made a good recovery. Later, at a fourth operation, Mr. Makins fixed the dilated loop, so that future twisting might be prevented, and she remains in good health.

A similar case is recorded by Richardson $:^{12}$

In this patient, a woman, the pelvic colon, which was so dilated as to fill the whole lower abdomen, was resected in 1899 and an anastomosis established between the iliac colon and a point in the pelvic colon below the dilated part. One year later, with recurrence of symptoms, it was found on exploration that a new dilated coil had developed in the place of the excised pelvic colon. Some adhesions which this new loop had contracted were separated without any improve loop Three months later a more complete exploration was made and it was found that "at the line of suture of the first resection, which made a straight passage from descending colon to rectum, a new coil bad appeared, around the central portion of which could be seen the scar of the suture line. Not only had a dilatation begun here, but it had bulged and spread until a new sigmoid flexure had appeared, and one so large that it the new verse plications we convexity of the new loop was reduced and its course became a moderate curve from the left pelvic brim to the rectum. No improvement resulted, so that about six months later Richardson resected the new dilated bowel and at the same time fixed the fundus of the uterus to the abdominal wall, in case it should be contributing to the dilatation by its pressure. At the time of writing it appears that the patient was still in trouble, and from the physical signs in the abdomen it seemed probable that a new dilated loop was in process of development for the second time.

My last case differs from the preceding in its anatomical features, but I think it belongs to the same group. Its peculiar character lies in the fact that the iliac and pelvic colon showed great muscular hypertrophy without any dilatation, and this pelvic part was short-in fact, nearly a straight passage from pelvic brim to rectum, and it was exceedingly rigid, and probably slightly narrowed. The whole of the rest of the colon was dilated without any hypertrophy. Still adhering to the supposition that all these colonic conditions have a nervous basis, I suppose that the explanation of this short hypertrophied portion is to be found in long-continued spasm. This case illustrates also the sudden collapse that often sets in on slight cause, and the generally precarious life of these patients.

CASE IX.-Defective Action of Colon from an early Age : Hypertrophy and Narrowing of Lower End: Dilatation without Hypertrophy above: Death.

Man, aged 48. He slipped and fractured his left patells. On admission into hospital it was noticed that he presented an odd appearance owing to the enormous size of the abdomen. On inquiry it was found that he had had difficulty in getting his bowels to act as far back as his memory went. In recent years, however, constipation had alternated with periods during wears, however, constipation had alternated with periods during which he passed five or six small liquid stools a day, as in.
Case viII, and his statement was that he found no remedy so efficient as beer.

There were some slight indications of intestinal coils to be seen through the abdominal wall, and slow alterations of shape were observed. Aperients and enemata produced no result, though flatus was passed. He was sick once. He sank rapidly, and died unrelieved six days after admission.

At the post-mortem examination it was found that there was great dilatation of the caecum, ascending transverse and descending parts of the colon. On opening the abdomen nothing but colon could be seen. The lower end of the descending colon, as it reached the iliac crest, was funnel-shaped and at this point the dilatation gradually ceased. To the naked eye there was no sign of muscular bypertrophy. The iliac and pelvic parts of the colon, however, were curiously short, lacking the usual curve, with great hypertrophy of the circular muscular coat, extreme rigidity, and, I think, distinct narrowing of the lumen. This part would just contain the index finger. (Fig. 9.) Microscopically no change was found anywhere in the colon, except the pure hypertrophy of muscle of the lower part. There was one shallow ulcer in the caecum of the lower part. There was one shallow ulcer in the caecum. The hypertrophy subsided at the lower end of the pelvic colon, and the rectum and anus were normal. The narrowed part of the colon was grey, the dilated part was reddish-grey, and the contrast in colour was distinct.

A case which has the same characters was recorded by Sir Frederick Treves : ${ }^{13}$

A girl, aged 6, presented a history of colon defect from birth "Two days after birth" vomiting began and continued off and on for several days, the bowels ceased to act, and the abdomen became distended-the story bearing an irresistible resemblance to that of Case IV, and of Dodd's and Osler's cases. Aperients had no effect, and relief was ultimately obtained by enemata. Similar attacks recurred at intervals, with increasing stipation and abdominal distension, until a permanent condition of dilatation of the colon was reached. Latterly 8 motion had only been obtained by passing a rigid tube 10 in. from the anus and then giving on injection. It was found on exploration that the ascending and transverse parts of the colon were nearly normal, though they had evidently been somewhat distended, and still showed some hypertrophy. The descending colon, however, was greatly dilated, while the rectum and sigmoid flexure were represented by a straight, solid-looking tube about the size of an adult forefinger, 8 in. or 
9 in. long, with no sacculation, but a very marked longitudinal muscular cost. He excised descending colon, sigmoid flexure, and upper part of rectum, effecting a junction between the left end of the transverse colon and the anus, with 8 perfect result and complete restoration of health.

\section{REFERENCES.}

1 Textbook of Anatomy. 2 Hale White, Allbutt's Syst. of Med., vol. ii Cheadle, Lancet, 1886, vol. ii. 4 Gee, St. Bart. Hosp. Rep., 1884.

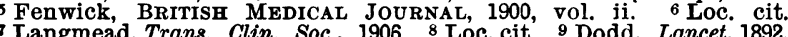
vol. i. 10 Osler, Jokns Hopkins Hosp. Bull. 1893, vol. iv 11 Clutton, Trans. Clin. Soc, 1897. 12 Richardson, Boston Med. and Surg. Journ., 1901. 13 Treves, Lancet, 1898, vol. i.

\section{NOTES ON A CASE OF ENTEROSPASM. \\ BY E. OLIVER ASHE, M.D.Lond., F.R.C.S.EnG., SURGEON TO KIMBERI.EY HOSPITAL.}

THE following case seems to be a typical example of "enterospasm," a disease about which very little is known, and of which I personally knew nothing at all, and failed to identify as a definitely recognized pathological condition at the time of the operation. Dr. Briggs, however, our junior house-surgeon, who saw the operation, said at once "that is the condition recently described in the British Medical Journal," and I find in the number for January 13th, 1906, a very careful and thorough account of a series of similar cases by Dr. Hawkins, of St. Thomas's.

The patient, a strong healthy woman, aged 22 , a member of a heal thy family well known to me, of active habits but inclined to be stout, first consulted me on March 22nd, 1906.

\section{History.}

She had been well and strong and had never ailed anythin until about December, 1905. She then began to be a little constipated, not always, but now and then, and to suffer from a feeling of discomfort in the left side of the stomach low down, which at times became actual pain, especially on walking far or fast. The pain did not last long and did not come frequently, sometimes staying away for a week. Early in January she got married, and was then apparently in perfect health. Whilst on her honeymoon at Johannesburg the pain and constipation became more troublesomesurg and phe pain and conaperients regularly, which she had never done before, and on her return home the constipation continued. On February 1st, her return home the constipation continued. On February 1st, seized with most acute abdominal pain accompanied by feeling of sickness, but she did not actually vomit. Various domestic remedies were tried to ease the pain, but gave no relief, so the nearest doctor was called in, and he gave a hypodermic of morphine which neither eased the pain nor sent her to sleep. Finding that the bowels had not acted satisfactorily for several days the doctor tried castor oil, cascara, Epsom salts, etc., and used enemas thoroughly, but in spite of all three days passed before the bowels were got to act, and during all this time the patient was in pain, fairly acute but not so acute as it had been at its first onset. After the bowels had acted well the pain passed off. The bowels were then kep open with an aperient containing cascara nux vomica and magnesium sulphate in rather large doses ; this had to be continued until I saw the patient at the end of March, though she was carefully dieted, and her manner of living was properly regulated.

\section{Condition while under Observation}

Examination of the abdo un was difficult on sccount of tenderness and a queer twitching of the muscles to which I shall refer later, but 1 failed to find any cause for the constipation, and considered the case to be one of atony of the bowel though this did not account for the acute attack of pain. prescribed a pill of aloin, belladonna, and nux vomica to be taken at bedtime. As this had no effect, I ordered in addition three-hourly doses of carcara and magnesium sulphate$1 \mathrm{drachm}$ of each-and this opened the bowels, but not at all freely.

On March 27th, the patient was put under chloroform, and a very careful and thorough examination was made-abdominal rectal, and vaginal-but nothing to account for the constipation could be found.

On March 29th a mixture containing extract of cascara sagrada liq. (B.P.) $\mathrm{ml}$, and tincture nux vomica $\mathrm{m} \mathrm{x}$, was substituted three times a day after food; to this as it failed to act properly, tincture of belladonns $m x$ were added on April 2nd, and; a daily enema of glycerine was found necessary on April 4th. On April 6th the belladonna was increased, but had no good result.

All this time there had been a good deal of tenderness and abdominal pain, not localized, worse when the bowels acted, and much worse when the enema was used; and though the bowels could only be got to act once a day, there was frequently a feeling that they were going to act, which had no result, no flatus even being passed, though there was rather severe straining.

The tenderness was curious, in that it was very markedly superficial ; if the hand was laid ever so lightly on any part of the abdomen, severe pain was felt at once, and an involuntar wincing or spasm of the abdominal muscles resulted, but this did not happen even on firm pressure over the lower ribs. This light pressure also gave rise to dcep pain, but my im pression was that the superficial pain was the worst; even when the patient was lying still in bed, this involuntary wincing kept happening without any apparent cause.

Belladonna and opium applications to the abdomen not in any way diminishing this excessive irritability, on April 13th the nux vomica was omitted from the medicine on the supposition that part of the irritability might be due to unusua susceptibility to strychnine, but no improvement in this noticed.

The glycerine enema now began to lose its effect, and a soap and water enema was used. On two occasions $I$ gave this myself, using a long tube (which, however, only could be got to pass about 4 in.) and a large quantity of water. On the second occasion I used about 4 pints of water, and, though given very slowly, this gave rise to most acute, in fact almost unbearable pain, accompanied with vomiting; $\frac{1}{4}$ gr. of morphine was given hypodermically, and was repeated in two hours, but did not seem to ease the pain in the least, which, however, subsided in a few hours.

During all this time the temperature was never raised, and there was never any abdominal distension, though the patient was positive that no flatus passed. Till the last week or so the patient was up and about; but when the large enema had to be used, owing to the failure of the glycerine to act, she had so much pain that she preferred to stay in bed. Finding that her condition was getting worse, that no medicine could be relied on to move the bowels, and that enemas were beginning to lose their effect, I decided, after consultation with Dr. Russell, to explore the abdomen.

My own opinion was that there was partial strangulation by a band, but as there was no history of any of the usual causes of band strangulation, such as peritonitis, tuberculous, general or pelvic, this was at best only a guess.

Operation.

The abdomen was accordingly opened below the umbilicus in the usual way on April 23rd. On introducing the hand something abnormal was felt almost immediately, just to the left of the incision. On bringing this out of the abdomen for examination, it proved to be a piece of small intestine very much narrowed. The narrowed portion was about 7 in. long and as thick as an ordinary finger, whilst the bowel above and and as thick as an ordinary finger, whilst the bowel above and below was the size of three fingers. There was no gradual tapering of the wide into the narrow part, or gradual expan-
sion of the narrow into the wide part again, but at one end of the narrow part the natural-sized bowel abruptly became contracted, and at the other resumed its natural calibre just as abruptly. There was no groove, twist, congestion, or sign of peritonitis to suggest that the bowel had been nipped or atrangulated in any way. The narrow part was firmly contracted, felt solid and fleshy, and was much paler than the rest of the bowel. It was to some extent pervious as flatus could be pressed through it from the adjoining bowel, but about be pressed through it from the adjoining bowel, but about a drachm of soft faecal matter was lodged in the normal bowel just at the beginning of the contracted part, and was evidently
unable to pass. Not being satisfied that all the symptoms were due to this contraction alone, the contracted part was covered with warm cloths and kept outside the abdomen while I tried to find some other cause for thom, but though I spent. ten minutes in very carefally examining the rest of the abdominal contents nothing else could be found. On then removing the cloths to look at the contracted part again, I found, to my intense surprise, that it had resumed its normal found, to my intense surprise, that it had resumed its normal size, feel, and appearance, and could only be distinguished
from the rest of the bowel by a slight difference in colour. The from the rest of the bowel by a slight difference in colour. The question how to treat the contracted part having thus solved stitched up in layers in the usual way.

The recovery after the operation was uneventful; a simple enema (one pint) was given on April 25th, the second morning after the operation, and, as the bowels acted quite well, it was continued every morning. On the evening of April 30th a pill of aloin, extract of belladonna, extract of nux vomica, blue pill, and capsicum was given, and acted so well that it was pill, and capsicum was given, and acted so well that it was repeated every night, and the enema was discontinued. On May 6th the twitching and abdominal tenderness which had been absent since the operation again returned, but in a much milder form, and only lasted a few days. On May 7 th the pill was reduced in strength, as it was acting quite well; on May 9th the bowels began to act too freely, and for three or four days acted five times a day, blood and mucus passing with the motion, but by May 14th constipation had again set in. Liquorice powder having no effect, the enema was again used. After experimenting with different aperients for a few days, After experimenting with different aperients for a few days, was therefore used each day with a good result.

On May 23rd, acting on Dr. Russell's suggestion, I ordered tincture of belladonna $m x$ three times a day after food, and this was increased until by Mav 30th the dose had reached mxx, and the full physiological effect had been obtained. The belladonina seemed to have very little effect, except that it allowed the glycerine enema to be reduced from 4 drachms to 2 drachms. The dose was therefore gradually diminished, and 